\title{
Riemann-Hilbert treatment of Liouville theory on the torus
}

\author{
Pietro Menotti \\ Dipartimento di Fisica, Università di Pisa and \\ INFN, Sezione di Pisa, Largo B. Pontecorvo 3, I-56127 \\ e-mail:menotti@df.unipi.it
}

October 2010

\begin{abstract}
We apply a perturbative technique to study classical Liouville theory on the torus. After mapping the problem on the cut-plane we give the perturbative treatment for a weak source. When the torus reduces to the square the problem is exactly soluble by means of a quadratic transformation in terms of hypergeometric functions. We give general formulas for the deformation of a torus and apply them to the case of the deformation of the square. One can compute the Heun parameter to first order and express the solution in terms of quadratures. In addition we give in terms of quadratures of hypergeometric functions the exact symmetric Green function on the square on the background generated by a one point source of arbitrary strength.
\end{abstract}




\section{Introduction}

Liouville theory has been widely studied both at the classical level (see e.g. [1, 2, 3, 4, 5]) and at the quantum level [5, 6, 7, 8, 9, 10, 11]. Most of the attention was devoted to the sphere and disk topologies [6, 7, $8,9,10$. Recently some notable progress have been performed for the torus topology [12, 13, 14, 15, 16].

Important results regarding the four point conformal correlation functions on the sphere and their relation to the one point function on the torus have been obtained [12]. Results related to conformal quantum field theory on the tours and to vortex theory on the torus are found in [17, 18] and [19, 20].

In [22, 23, 24] the sphere and the disk topologies were studied within the standard functional formulation of quantum field theory, i.e. by first computing a classical background and then integrating on the fluctuations about it. The three point case on the sphere, the one point case on the disk and also the two point case on the disk when one source is weak were studied in [22, 23, 24].

In this way it was possible to confirm the first few terms given by the bootstrap approach [6, 17, 8, 10] and also to compute some higher-point correlation functions when one source is weak [23]. The classical four point case on the sphere was studied in [22] when one of the sources is weak, in which case the problem is soluble by quadratures and as a byproduct one obtains the exact Green function on the background of three sources of arbitrary strength, provided they satisfy Picard's inequalities.

In this paper we shall consider Liouville theory at the classical level on the torus topology first in the general case and then we shall specialize to the one point source at the classical level. Even this simple configuration is not trivial. The case of the sphere, due to Picard inequalities, is meaningful only when the sources are at least three and the three point case is soluble in terms of hypergeometric functions through a well known procedure [5, 22. The simplicity of the solution of the three point case on the sphere is due to the fact that the accessory parameters are completely fixed by the Fuchs conditions and on the sphere the monodromy matrices are completely known [25, 5, 22, in terms of gamma-functions. On the torus through a transformation involving the Weierstrass $\wp$-function it is possible [26, 25, 12] to map the problem on the cut-plane but already with one point source the Fuchsian differential equation has four singularities and thus it becomes a special case of the Heun equation. A large literature exists on the Heun equation (see e.g. [27, 28, 29, 30]). The main problem in dealing with the 1-point problem on the torus is the determination of the Heun parameter (accessory parameter). Here the Fuchs conditions are not sufficient and the Heun parameter, which we shall call $\beta$ has to be determined along with an 
other parameter $k$ which also enters the 3-point solution on the sphere, by imposing the monodromic nature of the solution. This is a variant of the XXI Hilbert problem [31, 32]. A further shortcoming is the lack of analytical expressions for the monodromy matrices of the solutions of the Heun equation [27, 30, 33].

Obviously the exact solution cannot be given in general in terms of familiar functions like the hypergeometric functions, but the knowledge of $\beta$ from the data of the problem would be a decisive step. After $\beta$ is known, the parameter $k$ is easily computed.

One exception is the square configuration where by symmetry reasons the accessory parameter vanishes. Then by a quadratic transformation the exact solution is expressible in terms of hypergeometric functions. One can apply a perturbative technique developed in 22 to work out the solution on a deformed square and on the square in which a symmetric distribution of weak sources is added.

The structure of the paper is the following: in Sec2 we give the general setting of the problem. In Sec 3 we report general relations between the periods and the root $e_{k}$ of a third order equation which will be useful in the following. In Sec4 we give the perturbative treatment of the one point function. A byproduct is a derivation of the Green function on the torus which is alternative to the standard one [34, 35, 36]. In Sec.5 we show at the non perturbative level how the degrees of freedom appearing in the differential equation are sufficient to realize the monodromic solution. In Sec 6 we give the treatment of the square in terms of hypergeometric functions. Secs 78 deal with the perturbation induced by a general deformation of the torus and its application to the deformation of the square. Finally in Sec 9 we compute in terms of quadratures the exact symmetric Green function on the square in the background generated by an arbitrary charge.

Here we bound ourselves the the classical aspects; the technique of the expansion of quantum Liouville theory around classical solutions (semiclassical expansion) was applied with success to the case of the sphere topology [22] and to the disk topology [23, 24] for computing various correlation functions.

\section{General setting}

We write Liouville equation on the torus with $N$ point sources in the form

$$
-\partial_{z} \partial_{\bar{z}} \phi+e^{\phi}=2 \pi \sum_{n} \eta_{n} \delta^{2}\left(z-z_{n}\right) .
$$

It will be useful for the following to define $\lambda_{n}=1-2 \eta_{n}$. The $\eta_{n}$ are subject to the Picard's inequalities

$$
\eta_{n}<\frac{1}{2}, \quad \text { or } \quad 0<\lambda_{n}
$$


and

$$
\sum_{n} \eta_{n}>0
$$

The first inequality is requested by the local finiteness of the area while the second is due to the Gauss-Bonnet theorem.

A direct transcription of the problem (1) into a linear differential equation obtained by developing the torus on the plane would generate an equation of the type

$$
y^{\prime \prime}+Q(z) y=0
$$

with $Q(z)$ containing an infinite number of poles due to the infinite images of the $n$ sources and this is not a Fuchsian problem.

One performs the change of variables [25, 26] $u=\wp(z)$, being $\wp$ the Weierstrass function related to the torus whose periods we shall denote by $2 \omega_{1}, 2 \omega_{2}$. The above transformation due to $\wp(z)=\wp(-z)$ gives a two valued map from the cut-plane $u$ to the fundamental region in the $z$-plane which describes the torus. The original Liouville equation is transformed into

$$
-\partial_{u} \partial_{\bar{u}} \varphi+e^{\varphi}=2 \pi \sum_{n} \eta_{n} \delta^{2}\left(u-u_{n}\right)+\frac{\pi}{2}\left(\delta^{2}\left(u-e_{1}\right)+\delta^{2}\left(u-e_{2}\right)+\delta^{2}\left(u-e_{3}\right)\right)
$$

where

$$
\varphi=\phi+\log \left(\frac{d z}{d u} \frac{d \bar{z}}{d \bar{u}}\right) .
$$

The points $e_{1}, e_{2}, e_{3}$ are the images in the $u$-plane of the points $\omega_{1}, \omega_{2}$ and $\omega_{3} \equiv \omega_{1}+\omega_{2}$ and are subject to the condition $e_{1}+e_{2}+e_{3}=0$. If one source in eq.(1) is placed at the origin, say $z_{0}=0$ such a source does not appear in eq.(2) because the transformation takes it to infinity and it is taken into account by the behavior of $\varphi$ at infinity as dealt with in eq.(10) below. The new sources appearing at $e_{1}, e_{2}, e_{3}$ are due to the Laplacian of eq.(2) acting on the logarithms appearing in the r.h.s. of eq.(3) where due to a well known relation

$$
\wp^{\prime 2}=4 \wp^{3}-g_{2} \wp-g_{3} \equiv 4\left(u-e_{1}\right)\left(u-e_{2}\right)\left(u-e_{3}\right) .
$$

We choose for $d z / d u$ the determination

$$
\frac{d z}{d u}=-\frac{1}{2 \sqrt{\left(u-e_{1}\right)\left(u-e_{2}\right)\left(u-e_{3}\right)}}
$$

where the square root appearing in the denominator is positive for large positive $u$. It is useful to choose the cuts in the $u$-plane as to have all the source singularities which lay in the fundamental region of the $z$-plane mapped onto first sheet of the $u$-plane. The 
shape of such cuts is very simple when one has one, two or three sources. It becomes in general more complicated for a higher number of sources but it always exists.

In the present paper we shall restrict to the case in which the sources are symmetrical w.r.t. the reflection $z \rightarrow-z$. In this case it is not necessary to distinguish among the two sheets in $u$. We shall devote an other paper to non symmetric situation. For symmetric sources the problem is invariant under the transformation $z \rightarrow-z$ because the boundary of the fundamental region is also invariant under such a transformation. This implies that also the solution is parity invariant i.e. $\phi(-x,-y)=\phi(x, y)$. In fact otherwise we shall have at least two solutions of the problem, while a result by Picard [1] (see also [3, 4]) says that the solution exists and is unique. Thus for a symmetric distribution of sources the solution will be a single valued function of $u$.

The standard procedure for solving eq.(2) is to notice that as a consequence of the relation

$$
\partial_{u} \partial_{\bar{u}} \varphi=c e^{\varphi}
$$

away from the sources we have

$$
\partial_{\bar{u}}\left(e^{\frac{\varphi}{2}} \partial_{u}^{2} e^{-\frac{\varphi}{2}}\right)=0
$$

i.e.

$$
e^{\frac{\varphi}{2}} \partial_{u}^{2} e^{-\frac{\varphi}{2}}
$$

is an analytic function of $u$, call it $-Q(u)$. Then the general form of $e^{-\frac{\varphi}{2}}$ is

$$
e^{-\frac{\varphi}{2}}=\bar{\chi}_{1}(\bar{u}) \psi_{1}(u)+\bar{\chi}_{2}(\bar{u}) \psi_{2}(u)
$$

where $\psi_{1}$ and $\psi_{2}$ are two linearly independent solutions of

$$
y^{\prime \prime}(u)+Q(u) y(u)=0 .
$$

Noticing that we have also

$$
\partial_{u}\left(e^{\frac{\varphi}{2}} \partial_{\bar{u}}^{2} e^{-\frac{\varphi}{2}}\right)=0
$$

and due to the reality of $e^{-\frac{\varphi}{2}}$

$$
e^{\frac{\varphi}{2}} \partial_{\bar{u}}^{2} e^{-\frac{\varphi}{2}}=-\bar{Q}(\bar{u})
$$

we find that also $\chi_{1}$ and $\chi_{2}$ are solutions of eq.(5). Being the $\chi_{j}$ combinations of the $\psi_{j}$ again due to the reality of $e^{-\frac{\varphi}{2}}$ we can rewrite eq.(4I) in the form

$$
e^{-\frac{\varphi}{2}}=\bar{\psi}_{j}(\bar{u}) H_{j k} \psi_{k}(u)
$$

with $H_{j k}$ hermitean matrix. $H$ can always be diagonalized by means of a unitary transformation to the form $\mu_{1} \bar{y}_{1} y_{1}+\mu_{2} \bar{y}_{2} y_{2}$, with $\mu_{1}, \mu_{2}$ real and by a further rescaling of the 
$y_{k}$ to one of the forms $\bar{y}_{1} y_{1} \pm \bar{y}_{2} y_{2}$. The case of both negative eigenvalues is excluded by the positivity of $e^{-\frac{\varphi}{2}}$.

Denoting with $w_{12}$ the constant Wronskian

$$
w_{12}=y_{1} y_{2}^{\prime}-y_{1}^{\prime} y_{2}
$$

it is easily seen by explicit computation that for the upper sign [37] away from the sources we have

$$
\partial_{u} \partial_{\bar{u}} \varphi=-2\left|w_{12}\right|^{2} e^{\varphi}
$$

while for the lower sign

$$
\partial_{u} \partial_{\bar{u}} \varphi=2\left|w_{12}\right|^{2} e^{\varphi}
$$

which is the case we are interested in and we need to choose $\left|w_{12}\right|^{2}=1 / 2$. Thus we have reached for the solution of eq.(2) the form

$$
e^{\varphi}=\frac{2\left|w_{12}\right|^{2}}{\left[\bar{y}_{1}(\bar{u}) y_{1}(u)-\bar{y}_{2}(\bar{u}) y_{2}(u)\right]^{2}}
$$

where introducing explicitely the Wronskian we relaxed the condition $\left|w_{12}\right|^{2}=1 / 2$. The sources appearing in eq.(2) require the behavior of $y_{k}(u)$ to be power-like at the singularities. It means that $Q(u)$ has to be a meromorphic function with only first and second order poles i.e. of the form

$$
\begin{aligned}
Q(u) & =\frac{3}{16}\left(\frac{1}{\left(u-e_{1}\right)^{2}}+\frac{1}{\left(u-e_{2}\right)^{2}}+\frac{1}{\left(u-e_{3}\right)^{2}}\right)+\frac{b_{1}}{2\left(u-e_{1}\right)}+\frac{b_{2}}{2\left(u-e_{2}\right)}+\frac{b_{3}}{2\left(u-e_{3}\right)} \\
& +\sum_{n>0}\left(\frac{1-\lambda_{n}^{2}}{4\left(u-u_{n}\right)^{2}}+\frac{\beta_{n}}{2\left(u-u_{n}\right)}\right)
\end{aligned}
$$

where the $b_{k}$ and $\beta_{n}$ are the accessory parameters and they are subject to the Fuchs conditions

$$
\begin{gathered}
b_{1}+b_{2}+b_{3}+\sum_{n>0} \beta_{n}=0 \\
\frac{9}{16}+\frac{b_{1}}{2} e_{1}+\frac{b_{2}}{2} e_{2}+\frac{b_{3}}{2} e_{3}+\sum_{n>0} \frac{\beta_{n}}{2} u_{n}+\sum_{n>0} \frac{1-\lambda_{n}^{2}}{4}=\frac{4-\lambda^{2}}{16}
\end{gathered}
$$

where $\lambda=1-2 \eta_{0}$ is given by the source at the origin in eq.(11). At infinity $Q(u)$ behaves like

$$
\frac{4-\lambda^{2}}{16 u^{2}}
$$

and it assures that the $\phi$ field of eq.(1) obtained from $\varphi$ has the source at the origin $2 \pi \delta^{2}(z) \eta_{0}$ with $\eta_{0}=(1-\lambda) / 2$. 
In the case of a single source placed at the origin the general solution of eqs.(9) gives for $Q(u)$

$$
\begin{aligned}
Q(u) & =\frac{1-\lambda^{2}}{16} \frac{u+\beta}{\left(u-e_{1}\right)\left(u-e_{2}\right)\left(u-e_{3}\right)} \\
& +\frac{3}{16}\left(\frac{1}{\left(u-e_{1}\right)^{2}}+\frac{1}{\left(u-e_{2}\right)^{2}}+\frac{1}{\left(u-e_{3}\right)^{2}}+\frac{2 e_{1}}{\left(e_{1}-e_{2}\right)\left(e_{3}-e_{1}\right)\left(u-e_{1}\right)}\right. \\
& \left.+\frac{2 e_{2}}{\left(e_{2}-e_{3}\right)\left(e_{1}-e_{2}\right)\left(u-e_{2}\right)}+\frac{2}{\left(e_{3}-e_{1}\right)\left(e_{2}-e_{3}\right)\left(u-e_{3}\right)}\right) .
\end{aligned}
$$

Thus we are left with a single accessory parameter $\beta$ which has to be determined by imposing the monodromicity of the solution. We notice that contrary to the sphere case with three singularities the simple Fuchs conditions leave one accessory parameter undetermined.

For the $\varphi$ appearing in eq.(7) to be solution of eq.(2) it must be monodromic around the singularities for which it is necessary and sufficient that under a tour around each singularity the pair of function $y_{1}, y_{2}$ undergoes an $S U(1,1)$ transformation.

\section{The periods of the torus}

In this section we summarize some facts about the periods of the torus [25] in relation to the roots of the equation

$$
4 u^{3}-g_{2} u-g_{3}=4\left(u-e_{1}\right)\left(u-e_{2}\right)\left(u-e_{3}\right)
$$

and what happens in the particular cases in which the fundamental region becomes a rectangle or a square. Without loss of generality we can set $\omega_{1}=$ real, $\omega_{1}>0$ and $\omega_{2}=\omega_{2}^{R}+i \omega_{2}^{I}$ with $\omega_{2}^{I}>0$. From eq.(12) we have

$$
e_{1}+e_{2}+e_{3}=0
$$

In the case of the rectangle we have $\omega_{1}=\omega_{1}^{R}$ and $\omega_{2}=i \omega_{2}^{I}$ and as a consequence

$$
\begin{aligned}
& g_{2} \equiv 60 \sum_{m n}^{\prime} \frac{1}{\left(2 m \omega_{1}+2 n \omega_{2}\right)^{4}}=\text { real } \\
& g_{3} \equiv 140 \sum_{m n}^{\prime} \frac{1}{\left(2 m \omega_{1}+2 n \omega_{2}\right)^{6}}=\text { real }
\end{aligned}
$$

where in the sums the term $m=n=0$ is excluded. The discriminant of eq.(12) is

$$
\Delta=g_{2}^{3}-27 g_{3}^{2}=16\left(e_{1}-e_{2}\right)^{2}\left(e_{2}-e_{3}\right)^{2}\left(e_{3}-e_{1}\right)^{2}
$$


and we have [26]

$$
\Delta=\wp^{\prime}(z) \wp^{\prime}\left(z+\omega_{1}\right) \wp^{\prime}\left(z+\omega_{2}\right) \wp^{\prime}\left(z+\omega_{1}+\omega_{2}\right) .
$$

As for $\omega_{1}$ real and $\omega_{2}$ pure imaginary the $\wp$ function is a real analytic function i.e. $\wp(\bar{z})=$ $\overline{\wp(z)}$ we have also $\wp^{\prime}(\bar{z})=\overline{\wp^{\prime}(z)}$. Choosing in eq.(16) $z=-\frac{\omega_{1}}{2}-\frac{\omega_{2}}{2}$ we reach the conclusion $\Delta \geq 0$. As $g_{2}$ and $g_{3}$ are real we have that one of the solution, say $e_{2}$ is real. Then if $e_{1}$ has a non zero imaginary part we have $e_{3}=\bar{e}_{1}$ and

$$
\Delta=16\left(e_{2}-e_{1}\right)^{2}\left(e_{2}-\bar{e}_{1}\right)^{2}\left(2 i \operatorname{Im}\left(e_{1}\right)\right)^{2}<0
$$

which violates $\Delta \geq 0$. Thus we have $e_{1}$ real and as a consequence $e_{3}$ real. The conclusion is that for the rectangle we have all $e_{j}$ real. In the case of the square i.e. $\omega_{2}=i \omega_{1}$ with $\omega_{1}$ real, it is easily shown from the expression (15) that $g_{3}=0$. Then say $e_{2}=0$ and as $e_{1}+e_{2}+e_{3}=0$ we have $e_{3}=-e_{1}$ and as we are in a special case of rectangle we also have $e_{1}$ real and $e_{3}=-e_{1}$.

The inverse function of $u=\wp(z)$ can be written as

$$
z(u)=\int_{u}^{\infty} \frac{d x}{2 \sqrt{\left(x-e_{1}\right)\left(x-e_{2}\right)\left(x-e_{3}\right)}} .
$$

For large positive $x$ the square root is defined as $+(x)^{3 / 2}$. As $z$ goes along the path $0, \omega_{1}$, $\omega_{1}+\omega_{2} \equiv \omega_{3}, \omega_{2}, u$ follows a path from $+\infty, e_{1}, e_{3}$ and $e_{2}$. When $u$ reaches $e_{1}, u$ must continue below the branch point so that $z$ moves upward in the complex plane and again when $u$ reaches $e_{3}$ it must continue below the branch point as to have $z$ moving to the left in the complex plane.

\section{Perturbative solution for the one point function}

In this section we shall consider the case of a single weak source at the origin, i.e. $\eta_{0}=$ $(1-\lambda) / 2$ small. One can similarly apply the technique to several weak sources.

For $\lambda=1$ it is possible to solve eq.(5) exactly. Two independent solutions are given by

$$
\begin{gathered}
y_{1}(u)=\left[\left(u-e_{1}\right)\left(u-e_{2}\right)\left(u-e_{3}\right)\right]^{\frac{1}{4}} \equiv \Pi(u) \\
y_{2}(u)=\Pi(u) Z(u)
\end{gathered}
$$

where

$$
Z(u) \equiv z(u)-\omega_{1}-\omega_{2}=z(u)-\omega_{3}
$$

so that $Z\left(e_{1}\right)=-\omega_{2}, Z\left(e_{3}\right)=0, Z\left(e_{2}\right)=-\omega_{1}$. 
The Wronskian is given by

$$
w_{12}=y_{1} y_{2}^{\prime}-y_{1}^{\prime} y_{2}=\Pi^{2}(u) Z^{\prime}(u)=-\frac{1}{2}
$$

where the determination of $\Pi(u)$ has been chosen so that

$$
\Pi^{2}(u)=\sqrt{\left(u-e_{1}\right)\left(u-e_{2}\right)\left(u-e_{3}\right)} .
$$

We shall solve eq.(5) perturbatively by writing the solution as $y_{j}+\delta y_{j}$

$$
\delta y_{j}^{\prime \prime}+Q_{0} \delta y_{j}=-q y_{j}
$$

where

$$
\begin{aligned}
Q_{0}(u) & =\frac{3}{16}\left(\frac{1}{\left(u-e_{1}\right)^{2}}+\frac{1}{\left(u-e_{2}\right)^{2}}+\frac{1}{\left(u-e_{3}\right)^{2}}+\frac{2 e_{1}}{\left(e_{1}-e_{2}\right)\left(e_{3}-e_{1}\right)\left(u-e_{1}\right)}\right. \\
& \left.+\frac{2 e_{2}}{\left(e_{2}-e_{3}\right)\left(e_{1}-e_{2}\right)\left(u-e_{2}\right)}+\frac{2 e_{3}}{\left(e_{3}-e_{1}\right)\left(e_{2}-e_{3}\right)\left(u-e_{3}\right)}\right)
\end{aligned}
$$

and $q(u)$

$$
q(u)=\frac{\varepsilon}{16} \frac{u+\beta}{\left(u-e_{1}\right)\left(u-e_{2}\right)\left(u-e_{3}\right)}
$$

with $\varepsilon=1-\lambda^{2}$. The Green function of the unperturbed equation is

$$
G(u, x)=\frac{1}{w_{12}}\left(\Theta(u, x) y_{1}(u) y_{2}(x)-\Theta(u, x) y_{2}(u) y_{1}(x)\right) .
$$

The perturbed solutions are

$$
y_{j}+\delta y_{j}
$$

with

$$
\begin{aligned}
& \delta y_{j}(u)=\int_{e_{3}}^{\infty} G(u, x) q(x) y_{j}(x) d x= \\
& =\frac{1}{w_{12}}\left(y_{1}(u) \int_{e_{3}}^{u} y_{2}(x) q(x) y_{j}(x) d x-y_{2}(u) \int_{e_{3}}^{u} y_{1}(x) q(x) y_{j}(x) d x\right) \\
& =\frac{1}{w_{12}}\left(y_{1}(u) I_{j 2}(u)-y_{2}(u) I_{j 1}(u)\right)
\end{aligned}
$$

with

$$
I_{j k}(u)=\int_{e_{3}}^{u} q(x) y_{j}(x) y_{k}(x) d x .
$$

Thus

$$
I_{11}(u)=\frac{\varepsilon}{16} \int_{e_{3}}^{u} \frac{(x+\beta) d x}{\sqrt{\left(x-e_{1}\right)\left(x-e_{2}\right)\left(x-e_{3}\right)}}
$$




$$
\begin{aligned}
& I_{12}(u)=\frac{\varepsilon}{16} \int_{e_{3}}^{u} \frac{(x+\beta) Z(x) d x}{\sqrt{\left(x-e_{1}\right)\left(x-e_{2}\right)\left(x-e_{3}\right)}}, \\
& I_{22}(u)=\frac{\varepsilon}{16} \int_{e_{3}}^{u} \frac{(x+\beta) Z^{2}(x) d x}{\sqrt{\left(x-e_{1}\right)\left(x-e_{2}\right)\left(x-e_{3}\right)}} .
\end{aligned}
$$

The accessory parameter $\beta$ will have to be fixed as to have that for a tour around each singularity $e_{k}$ and infinity the $\varphi$ appearing in eq.(17) be monodromic.

At $e_{3}$ the behavior of the $I_{j k}$ is the following

$$
I_{11}(u)=\left(u-e_{3}\right)^{\frac{1}{2}} f_{11}\left(u-e_{3}\right), \quad I_{12}(u)=\left(u-e_{3}\right) f_{12}\left(u-e_{3}\right), \quad I_{22}(u)=\left(u-e_{3}\right)^{\frac{3}{2}} f_{22}\left(u-e_{3}\right)
$$

where $f_{j k}$ and the $f_{j}$ immediately below denote power series in $u-e_{3}$. Combining with the behavior of $y_{j}$ at $e_{3}$ we have that

$$
\delta y_{1}=\left(u-e_{3}\right)^{\frac{5}{4}} f_{1}\left(u-e_{3}\right), \quad \delta y_{2}=\left(u-e_{3}\right)^{\frac{7}{4}} f_{2}\left(u-e_{3}\right)
$$

so that the monodromic behavior of the perturbed functions at $e_{3}$ is unchanged independently of the value of $\beta$. Notice that the Wronskian $w_{12}$ is left unchanged by the perturbation.

We examine now the monodromic properties at $e_{1}$. With regard to $y_{1}=\Pi(u)$ the expression $\bar{y}_{1} y_{1}$ is monodromic around $e_{1}$. Instead for $y_{2}=\Pi(u) Z(u)$ we have for $Z(u)$ around $e_{1}$

$$
\begin{aligned}
Z(u) & \approx \frac{1}{2} \int_{e_{1}}^{e_{3}} \frac{d x}{\sqrt{\left(x-e_{1}\right)\left(x-e_{2}\right)\left(x-e_{3}\right)}}-\frac{\left(u-e_{1}\right)^{\frac{1}{2}}}{\sqrt{\left(e_{1}-e_{2}\right)\left(e_{1}-e_{3}\right)}}= \\
& =-\omega_{2}-\frac{\left(u-e_{1}\right)^{\frac{1}{2}}}{\sqrt{\left(e_{1}-e_{2}\right)\left(e_{1}-e_{3}\right)}}
\end{aligned}
$$

Then

$$
y_{2} \approx \Pi(u)\left[-\omega_{2}-s_{1}\left(u-e_{1}\right)^{\frac{1}{2}}\right]
$$

where

$$
s_{1}=\frac{1}{\sqrt{\left(e_{1}-e_{2}\right)\left(e_{1}-e_{3}\right)}} .
$$

Thus $\bar{y}_{2} y_{2}$ is not monodromic at $e_{1}$. 
We examine now $\delta y_{1}$ around $e_{1}$.

$$
\begin{aligned}
\delta y_{1} & =\frac{\Pi(u)}{w_{12}}\left[I_{12}\left(e_{1}\right)+\frac{\varepsilon}{16} \int_{e_{1}}^{u} \frac{(x+\beta) Z(x) d x}{\sqrt{\left(x-e_{1}\right)\left(x-e_{2}\right)\left(x-e_{3}\right)}}\right. \\
& \left.-Z(u) \frac{\varepsilon}{16} \int_{e_{3}}^{u} \frac{(x+\beta) d x}{\sqrt{\left(x-e_{1}\right)\left(x-e_{2}\right)\left(x-e_{3}\right)}}\right] \\
& \approx \frac{\Pi(u)}{w_{12}}\left[I_{12}\left(e_{1}\right)+\frac{\varepsilon}{16} 2 s_{1}\left(e_{1}+\beta\right) Z\left(e_{1}\right)\left(u-e_{1}\right)^{\frac{1}{2}}\right. \\
& \left.-\left(Z\left(e_{1}\right)-s_{1}\left(u-e_{1}\right)^{\frac{1}{2}}\right)\left(I_{11}\left(e_{1}\right)+\frac{\varepsilon}{16} 2 s_{1}\left(e_{1}+\beta\right)\left(u-e_{1}\right)^{\frac{1}{2}}\right)\right]
\end{aligned}
$$

i.e.

$$
y_{1}+\delta y_{1}=\Pi(u)\left[1+\varepsilon \text { const }+\frac{s_{1} I_{11}\left(e_{1}\right)\left(u-e_{1}\right)^{\frac{1}{2}}}{w_{12}}\right] .
$$

Then we must impose the monodromicity of

$$
\mid\left.\Pi(u)\left(1+\varepsilon \text { const }+\frac{s_{1} I_{11}\left(e_{1}\right)\left(u-e_{1}\right)^{\frac{1}{2}}}{w_{12}}\right)\right|^{2}-|k|^{2}\left|\Pi(u)\left(-\omega_{2}-s_{1}\left(u-e_{1}\right)^{\frac{1}{2}}\right)\right|^{2}
$$

where being already $|k|^{2}$ of first order in $\varepsilon$ we have neglected the correction $\delta y_{2}$ to $y_{2}$.

To order $\varepsilon$, taking into account that the $I_{j k}$ are themselves of order $\varepsilon$, the monodromicity of the previous expression becomes

$$
0=\frac{I_{11}\left(e_{1}\right) s_{1}\left(u-e_{1}\right)^{\frac{1}{2}}}{w_{12}}-|k|^{2} \bar{\omega}_{2} s_{1}\left(u-e_{1}\right)^{\frac{1}{2}}
$$

or

$$
0<|k|^{2}=\frac{I_{11}\left(e_{1}\right)}{w_{12} \bar{\omega}_{2}}
$$

$I_{11}\left(e_{1}\right)$ can be explicitely computed because we have

$$
\begin{aligned}
I_{11}\left(e_{1}\right) & =\frac{\varepsilon}{16} \int_{e_{3}}^{e_{1}} \frac{(\beta+x) d x}{\sqrt{\left(x-e_{1}\right)\left(x-e_{2}\right)\left(x-e_{3}\right)}}=\frac{\varepsilon}{8}\left[\beta\left(z\left(e_{3}\right)-z\left(e_{1}\right)\right)-\int_{z\left(e_{3}\right)}^{z\left(e_{1}\right)} \wp(z) d z\right] \\
& =\frac{\varepsilon}{8}\left[\beta \omega_{2}+\zeta\left(\omega_{1}\right)-\zeta\left(\omega_{1}+\omega_{2}\right)\right]=\frac{\varepsilon}{8}\left[\beta \omega_{2}-\zeta\left(\omega_{2}\right)\right]
\end{aligned}
$$

where we used the property of the $\zeta$ function [25]

$$
\zeta^{\prime}(z)=-\wp(z)
$$

and we know that 25$]$

$$
\zeta\left(z+2 \omega_{j}\right)=\zeta(z)+2 \zeta\left(\omega_{j}\right), \quad \zeta\left(\omega_{1}+\omega_{2}\right)=\zeta\left(\omega_{1}\right)+\zeta\left(\omega_{2}\right) .
$$


Similarly we have, working around the point $e_{2}$

with

$$
0<|k|^{2}=\frac{I_{11}\left(e_{2}\right)}{w_{12} \bar{\omega}_{1}}
$$

$$
I_{11}\left(e_{2}\right)=\frac{\varepsilon}{8}\left[\beta \omega_{1}-\zeta\left(\omega_{1}\right)\right] .
$$

Solving the system of eqs.(36],39) we have

$$
\beta=\frac{\zeta\left(\omega_{2}\right) \bar{\omega}_{1}-\zeta\left(\omega_{1}\right) \bar{\omega}_{2}}{\omega_{2} \bar{\omega}_{1}-\omega_{1} \bar{\omega}_{2}}
$$

and

$$
|k|^{2}=\frac{\varepsilon\left(\omega_{2} \zeta\left(\omega_{1}\right)-\omega_{1} \zeta\left(\omega_{2}\right)\right)}{8 w_{12}\left(\omega_{1} \bar{\omega}_{2}-\omega_{2} \bar{\omega}_{1}\right)} .
$$

Using now Legendre relation 25 ]

$$
\omega_{2} \zeta\left(\omega_{1}\right)-\omega_{1} \zeta\left(\omega_{2}\right)=\frac{\pi i}{2}
$$

which holds for $\operatorname{Im}\left(\frac{\omega_{2}}{\omega_{1}}\right)>0$ which is our case, we have

$$
|k|^{2}=\frac{\varepsilon \pi}{4 A}
$$

being $A$ the area of the fundamental region. We see that such a relation can be satisfied with $|k|^{2}>0$ only for $\varepsilon>0$ i.e. $\eta>0$ in accordance with the Gauss-Bonnet theorem. The quadratures appearing in $\delta y_{1}(u)$ i.e. the $I_{12}(u), I_{11}(u)$ can all be done in terms of elliptic functions. Using eq.(38) and [25]

$$
\zeta(z)=\frac{\zeta\left(\omega_{1}\right)}{\omega_{1}} z+\frac{\theta_{1}^{\prime}(v \mid \tau)}{2 \omega_{1} \theta_{1}(v \mid \tau)}, \quad \text { with } \quad v=\frac{z}{2 \omega_{1}} \text { and } \tau=\frac{\omega_{2}}{\omega_{1}}
$$

we obtain, making use again of the Legendre relation,

$$
\begin{aligned}
& e^{-\frac{\phi}{2}}=\frac{1}{\sqrt{2}|k|}\left(1+\frac{\varepsilon}{4}\left[\left(\log \frac{\theta_{1}\left(\frac{z}{2 \omega_{1}} \mid \tau\right)}{\theta_{1}\left(\frac{\omega_{3}}{2 \omega_{1}} \mid \tau\right)}+c . c .\right)-\right.\right. \\
& \left.\left.\frac{i \pi}{4\left(\omega_{1} \bar{\omega}_{2}-\bar{\omega}_{1} \omega_{2}\right)}\left(\frac{\bar{\omega}_{1}}{\omega_{1}} z^{2}+\frac{\omega_{1}}{\bar{\omega}_{1}} \bar{z}^{2}-2 z \bar{z}\right)+\frac{i \pi}{\omega_{1} \bar{\omega}_{1}}\left(\omega_{1} \bar{\omega}_{2}-\bar{\omega}_{1} \omega_{2}\right)\right]\right) .
\end{aligned}
$$

The derivative of $\phi$ with respect to $\varepsilon$ obviously provides the Green function on the flat torus, which is defined up to an additive constant. One obtains with the standard values $2 \omega_{1}=1$ and $2 \omega_{2}=\tau^{R}+i \tau^{I}$

$$
G(z)=\frac{1}{4 \pi} \log \left|\theta_{1}(z \mid \tau)\right|^{2}+\frac{1}{8 \tau^{I}}(z-\bar{z})^{2}
$$

satisfying

$$
\Delta G(z)=\delta(z)-\frac{1}{\tau^{I}}
$$

Equation (42) was derived in [34, 35, 36] by summing the Fourier representation of the Green function in momentum space by using Kronecker resummation formula [36]. Here it has been derived by a quadrature. 


\section{Counting the degrees of freedom}

The above perturbative calculation raises the following general question which we want to investigate at the non perturbative level. We have to satisfy the monodromy of $\varphi$ at $e_{1}$, $e_{2}$ having at our disposal only the three real parameters $\beta^{R}, \beta^{I}$ and $|k|^{2}$ the last of which has to result positive. Monodromy of $\varphi$ means that the monodromy matrices $M\left(e_{k}\right)$ at $e_{1}$ and $e_{2}$ belong to $S U(1,1)$, being this a necessary and sufficient condition for monodromy and three real parameters appear too few to satisfy the four complex equations for the matrix elements $M_{22}\left(e_{k}\right)=\bar{M}_{11}\left(e_{k}\right), M_{21}\left(e_{k}\right)=\bar{M}_{12}\left(e_{k}\right)$ with $k=1,2$.

We choose as before $y_{k}$ to be canonical at $e_{3}$ i.e. $y_{1}=\left(u-e_{3}\right)^{\frac{1}{4}} f_{1}\left(u-e_{3}\right), \quad y_{2}=$ $\left(u-e_{3}\right)^{\frac{3}{4}} f_{2}\left(u-e_{3}\right)$. If we denote by $S$ the $S L(2, C)$ transformation which expresses the solution canonical at $e_{3}$

$$
Y=\left(\begin{array}{l}
y_{1} \\
y_{2}
\end{array}\right)
$$

around $e_{1}$ as

$$
Y=S\left(\begin{array}{l}
\zeta^{\frac{1}{4}} g_{1}(\zeta) \\
\zeta^{\frac{3}{4}} g_{2}(\zeta)
\end{array}\right)
$$

with $\zeta=u-e_{1}$ and

$$
S=\left(\begin{array}{ll}
a & b \\
c & d
\end{array}\right)
$$

we have for the monodromy matrix at $e_{1}$

$$
M=i\left(\begin{array}{cc}
a d+b c & -2 a b \\
2 c d & -a d-b c
\end{array}\right) .
$$

We can also multiply $Y$ by the matrix

$$
K=\left(\begin{array}{cc}
\kappa^{-1} & 0 \\
0 & \kappa
\end{array}\right)
$$

without altering its monodromy property at $e_{3}$. Then we find for the monodromy matrix at $e_{1}$

$$
M=\left(\begin{array}{cc}
i(a d+b c) & -2 i a b \kappa^{-2} \\
2 i c d \kappa^{2} & -i(a d+b c)
\end{array}\right)
$$

i.e. we have at all the points $e_{k}$

$$
1=M_{11} M_{22}-M_{12} M_{21} \quad \text { and } \quad M_{11}+M_{22}=0
$$

independently of the imposition of the monodromy. 
Imposition of the $S U(1,1)$ nature of $M$ gives

$$
a b \kappa^{-2}=\bar{c} \bar{d} \bar{\kappa}^{2} \quad \text { i.e. } \quad \kappa^{2} \bar{\kappa}^{2}=\frac{a b}{\bar{c} \bar{d}} .
$$

This is sufficient also to give $M_{22}=\overline{M_{11}}$ because we have

$$
a b c d=\text { real }>0, \quad \text { i.e. } \quad a d=r_{1} \theta, \quad b c=r_{2} \bar{\theta}, \quad r_{1} \geq 0, \quad r_{2} \geq 0
$$

with $\theta$ a unitary number, which combined with

$$
a d-b c=1=r_{1} \theta-r_{2} \bar{\theta}
$$

gives

$$
\left(r_{1}+r_{2}\right)(\theta-\bar{\theta})=0, \quad \text { i.e. } \quad a d=\text { real, } \quad b c=\text { real } .
$$

We can spend now the complex parameter $\beta=\beta^{R}+i \beta^{I}$ for solving the complex equation

$$
\frac{a b}{\bar{c} \bar{d}}\left(e_{1}\right)=\frac{a b}{\bar{c} \bar{d}}\left(e_{2}\right)
$$

and we are left with proving the reality and positivity of such a ratio.

At the perturbative level we saw that Legendre relation solves this problem by giving to $\kappa^{2} \bar{\kappa}^{2}$ a real positive value as it must be. We want to understand here this problem at the nonperturbative level.

The monodromy matrix at $e_{3}$ where the solutions are canonical with indices $1 / 4,3 / 4$ is given by

$$
M\left(e_{3}\right)=D(0)=\left(\begin{array}{cc}
i & 0 \\
0 & -i
\end{array}\right),
$$

and we have the group relation

$$
D(0) M\left(e_{2}\right) M\left(e_{1}\right)=M^{-1}(\infty)
$$

and by construction all matrices are of $S L(2, C)$ type. Using eq.(43) we can write

$$
M\left(e_{2}\right)=\left(\begin{array}{cc}
m_{11} & r_{1} e^{i \phi_{1}} \\
r_{2} e^{i \phi_{2}} & -m_{11}
\end{array}\right), \quad M\left(e_{1}\right)=\left(\begin{array}{cc}
n_{11} & s_{1} e^{i \psi_{1}} \\
s_{2} e^{i \psi_{2}} & -n_{11}
\end{array}\right) .
$$

We have

$$
\operatorname{Tr}\left(D(0) M\left(e_{2}\right) M\left(e_{1}\right)\right)=i\left[\left(M\left(e_{2}\right) M\left(e_{1}\right)\right)_{11}-\left(M\left(e_{2}\right) M\left(e_{1}\right)\right)_{22}\right]=-2 \cos \frac{\pi \lambda}{2}=\text { real }
$$

which becomes

$$
i\left(r_{1} s_{2} e^{i\left(\phi_{1}+\psi_{2}\right)}-r_{2} s_{1} e^{i\left(\phi_{2}+\psi_{1}\right)}\right)=\text { real }
$$


or

$$
r_{1} s_{2} \cos \left(\phi_{1}+\psi_{2}\right)-r_{2} s_{1} \cos \left(\phi_{2}+\psi_{1}\right)=0
$$

As already mentioned we can spend $\beta^{R}+i \beta^{I}$ to satisfy eq.(44) i.e. $r_{1} / r_{2}=s_{1} / s_{2}$ and $e^{i\left(\phi_{1}+\phi_{2}\right)}=e^{i\left(\psi_{1}+\psi_{2}\right)}$. Thus

$$
\phi_{1}+\phi_{2}=\psi_{1}+\psi_{2}(\bmod 2 \pi) \text {. }
$$

We cannot have for the solution of (50)

$$
\phi_{1}+\psi_{2}=\psi_{1}+\phi_{2}(\bmod 2 \pi)
$$

otherwise $\operatorname{TrM}(\infty)=0$ for the $\beta$ which realizes eq.(44) against eq.(48). Then

$$
\phi_{1}+\psi_{2}=-\psi_{1}-\phi_{2}(\bmod 2 \pi), \quad \text { or } \quad \phi_{1}+\phi_{2}=-\psi_{1}-\psi_{2} \quad(\bmod 2 \pi)
$$

which combined with (151) gives

$$
\psi_{2}=-\psi_{1}(\bmod 2 \pi), \quad \phi_{2}=-\phi_{1}(\bmod 2 \pi)
$$

and we have a $\kappa$ which renders $M\left(e_{3}\right), M\left(e_{2}\right), M\left(e_{1}\right)$, and thus also $M(\infty)$ of $S U(1,1)$ type. The results of [1, 3, 4] tell us that when Picard's inequalities are satisfied, such $\beta$ exists in agreement with the perturbative result.

\section{The square}

We shall now solve at the non perturbative level a special case, i.e. the one in which the fundamental region of the torus is a square. In such a situation the function $Q(u)$ becomes with $e_{3}=0, e_{1}=-e_{2}=1$ (see Sec $\left.\sqrt[3]{ }\right)$

$$
Q(u)=\frac{\left(1-\lambda^{2}\right)(u+\beta)}{16 u\left(u^{2}-1\right)}+\frac{3}{16} \frac{\left(1+u^{2}\right)^{2}}{u^{2}\left(1-u^{2}\right)^{2}} .
$$

The following formal argument supports the result $\beta=0$ for the square. Suppose the monodromy is achieved for a certain value of $\beta$. We can now perform the transformation $u \rightarrow-u$ which is equivalent to $\beta \rightarrow-\beta$. Thus if $\beta$ is a solution also $-\beta$ is, and if the solution is unique we have $\beta=0$. We shall prove explicitely in the following that the differential equation with $\beta=0$ solves all the monodromy conditions.

Setting $\beta=0$ we have

$$
Q(u)=\frac{1-\lambda^{2}}{16\left(u^{2}-1\right)}+\frac{3}{16} \frac{\left(1+u^{2}\right)^{2}}{u^{2}\left(1-u^{2}\right)^{2}}
$$


Using $x=u^{2}$ we have

$$
4 x \frac{d^{2} y}{d x^{2}}+2 \frac{d y}{d x}+\left[\frac{1-\lambda^{2}}{16(x-1)}+\frac{3}{16} \frac{(1+x)^{2}}{x(1-x)^{2}}\right] y=0
$$

giving rise to the following $P$-symbol

$$
P\left(\begin{array}{ccc}
0 & \infty & 1 \\
3 / 8 & -1 / 4+\lambda / 8 & 3 / 4 \\
1 / 8 & -1 / 4-\lambda / 8 & 1 / 4
\end{array}\right)
$$

Two independent solutions canonical at $e_{3}=0$ are

$$
\begin{aligned}
& y_{1}=u^{\frac{1}{4}}\left(1-u^{2}\right)^{\frac{1}{4}} F\left(\frac{1+\lambda}{8}, \frac{1-\lambda}{8} ; \frac{3}{4} ; u^{2}\right) \\
& y_{2}=u^{\frac{3}{4}}\left(1-u^{2}\right)^{\frac{1}{4}} F\left(\frac{3+\lambda}{8}, \frac{3-\lambda}{8} ; \frac{5}{4} ; u^{2}\right) .
\end{aligned}
$$

The analytic continuation at $u^{2}=1$ is given by [25]

$$
\begin{gathered}
y_{1}=u^{\frac{1}{4}}\left(1-u^{2}\right)^{\frac{1}{4}}\left[A_{1} F\left(a, b ; a+b-c+1 ; 1-u^{2}\right)+\right. \\
\left.A_{2}\left(1-u^{2}\right)^{c-a-b} F\left(c-a, c-b ; c-a-b+1 ; 1-u^{2}\right)\right] \\
y_{2}=u^{\frac{3}{4}}\left(1-u^{2}\right)^{\frac{1}{4}}\left[A_{1}^{\prime} F\left(a^{\prime}, b^{\prime} ; a^{\prime}+b^{\prime}-c^{\prime}+1 ; 1-u^{2}\right)+\right. \\
\left.A_{2}^{\prime}\left(1-u^{2}\right)^{c^{\prime}-a^{\prime}-b^{\prime}} F\left(c^{\prime}-a^{\prime}, c^{\prime}-b^{\prime} ; c^{\prime}-a^{\prime}-b^{\prime}+1 ; 1-u^{2}\right)\right]
\end{gathered}
$$

with

$$
A_{1}=\frac{\Gamma(c) \Gamma(c-a-b)}{\Gamma(c-a) \Gamma(c-b)}, \quad A_{2}=\frac{\Gamma(c) \Gamma(a+b-c)}{\Gamma(a) \Gamma(b)}
$$

giving

$$
|\kappa|^{4}=\frac{a b}{\bar{c} \bar{d}}=\left(\frac{\Gamma(3 / 4)}{\Gamma(5 / 4)}\right)^{2} \frac{\Gamma\left(\frac{3+\lambda}{8}\right) \Gamma\left(\frac{3-\lambda}{8}\right) \Gamma\left(\frac{7+\lambda}{8}\right) \Gamma\left(\frac{7-\lambda}{8}\right)}{\Gamma\left(\frac{1-\lambda}{8}\right) \Gamma\left(\frac{1+\lambda}{8}\right) \Gamma\left(\frac{5+\lambda}{8}\right) \Gamma\left(\frac{5-\lambda}{8}\right)} .
$$

This is the non perturbative value of $|\kappa|^{4}$; for small $\varepsilon \equiv 1-\lambda^{2} \approx 2(1-\lambda)$ we have

$$
|\kappa|^{4}=\frac{a b}{\bar{c} \bar{d}} \approx\left(\frac{\Gamma(3 / 4)}{\Gamma(5 / 4)}\right)^{2} \frac{1}{\Gamma\left(\frac{\varepsilon}{16}\right)} \approx\left(\frac{\Gamma(3 / 4)}{\Gamma(5 / 4)}\right)^{2} \frac{\varepsilon}{16} .
$$

This is in agreement with the perturbative calculation given previously

$$
|\kappa|^{4}=\frac{\varepsilon \pi}{4 A}
$$

as in our case we have $g_{2}=4, g_{3}=0$ and

$$
\omega_{1}=-i \omega_{2}=\frac{1}{2} \int_{1}^{\infty} \frac{d u}{\sqrt{u^{3}-u}}=\frac{\sqrt{\pi} \Gamma(5 / 4)}{\Gamma(3 / 4)}=1.31103 \ldots
$$


Taking into account eqs.(37) we have for the square torus

$$
e^{-\frac{\phi(z)}{2}}=\frac{1}{\sqrt{2}|\kappa|^{2}}\left[\left|F\left(\frac{1+\lambda}{8}, \frac{1-\lambda}{8} ; \frac{3}{4} ; u^{2}(z)\right)\right|^{2}-|\kappa|^{4}|u(z)|\left|F\left(\frac{3+\lambda}{8}, \frac{3-\lambda}{8} ; \frac{5}{4} ; u^{2}(z)\right)\right|^{2}\right]
$$

where $|\kappa|^{4}$ is given by eq. (63).

From the derivative of $\phi$, given by eq.(65), with respect to $\varepsilon$ for $\varepsilon=0$ we obtain the Green function for the torus given by the square with half periods $\omega_{1}=-i \omega_{2}$ related to the invariants we are working with i.e. $g_{2}=4, g_{3}=0\left(\omega_{1}=-i \omega_{2}=1.31103 \ldots\right)$

$$
G_{s}(z)=\frac{1}{16 \pi}\left(2 \operatorname{Re}\left(F^{(1)}\left(0, \frac{1}{4} ; \frac{3}{4} ; u^{2}(z)\right)\right)-\left(\frac{\Gamma\left(\frac{3}{4}\right)}{\Gamma\left(\frac{5}{4}\right)}\right)^{2}|u(z)|\left|F\left(\frac{1}{4}, \frac{1}{2} ; \frac{5}{4} ; u^{2}(z)\right)\right|^{2}\right)
$$

where $F^{(1)}$ denotes the derivative of the hypergeometric function with respect to the first argument. It gives an expression for the Green function on the square alternative to eq.(42). The relation of $G_{s}$ to eq.(42) is

$$
G_{s}\left(2 \omega_{1} z\right)=\frac{1}{4 \pi} \log \left|\theta_{1}(z \mid i)\right|^{2}+\frac{1}{8}(z-\bar{z})^{2}+\text { const. }
$$

\section{General deformation}

We shall develop here the formulas for a deformation starting from a general configuration; later we shall apply it to the case of the square.

To the half-periods $\omega_{1}, \omega_{2}$ there corresponds according to the formulas reported in Sec 3 the roots $e_{1}, e_{2}, e_{3}$ subject to the restriction (13). It is however simpler in working with the differential equation to work with singularities whose locations are not restricted by (13). The original situation can be recovered by a simple translation.

We shall choose as position of the singularities in the $u$-plane $-1,0$ and $u_{1}$. A general change in $u_{1}$ has two real degrees of freedom which correspond to the changes in $\omega_{2} / \omega_{1}$. The remaining transformations in the periods are trivial i.e. the rotations and the dilatations. Again we shall choose two unperturbed solutions which are canonical at 0 and then impose the monodromy requirements in -1 and $u_{1}$. The first problem is rather simple. The maintenance of the monodromy at the moving singularity $u_{1}$ is rather complicated and we shall avoid it by a proper coordinate transformation.

The $Q$ becomes

$$
\begin{aligned}
Q(u)= & \frac{h}{16} \frac{u+\beta}{u(u+1)\left(u-u_{1}\right)}+ \\
& \frac{3}{16}\left(\frac{1}{\left(u-u_{1}\right)^{2}}+\frac{1}{u^{2}}+\frac{1}{(u+1)^{2}}-\frac{2}{\left(u_{1}+1\right)\left(u-u_{1}\right)}+\frac{2}{\left(u_{1}+1\right)(u+1)}\right)
\end{aligned}
$$


and under the perturbation $u_{1} \rightarrow u_{1}+\varepsilon$ we have the variation of $Q(u)$

$$
\begin{aligned}
q(u)= & \varepsilon\left[\frac{h\left(u^{2}-\beta u_{1}\right)+u\left(6+\beta h+6 u_{1}-h u_{1}\right)}{16 u(1+u)\left(u-u_{1}\right)^{3}}\right]+ \\
& \beta_{1} \frac{h}{16 u(u+1)\left(u-u_{1}\right)} .
\end{aligned}
$$

We now denote with $y_{1}(u), y_{2}(u)$ the two solutions, canonical at $u=0$ which behaves respectively as $u^{1 / 4}$ and $u^{3 / 4}$ whose Wronskian is $1 / 2$. The behavior of $y_{k}(u)$ around $u=-1$ will be

$$
\begin{aligned}
& y_{1}(u) \approx a \zeta^{1 / 4}+b \zeta^{3 / 4} \\
& y_{2}(u) \approx c \zeta^{1 / 4}+d \zeta^{3 / 4}
\end{aligned}
$$

with $\zeta=u+1$ and

$$
\left(\begin{array}{ll}
a & b \\
c & d
\end{array}\right)
$$

an $S L(2, C)$ matrix. Using the same notation as in Sec 4 for the variation of the solutions we have the behavior around $u=-1$

$$
\begin{aligned}
\delta y_{1}(u) \approx & \frac{a \zeta^{1 / 4}+b \zeta^{3 / 4}}{w_{12}}\left[I_{12}(-1)+\int_{-1}^{u} q(x) y_{1}(x) y_{2}(x) d x\right] \\
- & \frac{c \zeta^{1 / 4}+d \zeta^{3 / 4}}{w_{12}}\left[I_{11}(-1)+\int_{-1}^{u} q(x) y_{1}(x) y_{1}(x) d x\right] \approx \\
& \frac{a \zeta^{1 / 4}+b \zeta^{3 / 4}}{w_{12}} I_{12}(-1)-\frac{c \zeta^{1 / 4}+d \zeta^{3 / 4}}{w_{12}} I_{11}(-1)
\end{aligned}
$$

the reason being that the terms of order $\zeta^{1 / 2}$ generated in the integrals from -1 to $u$ cancel, leaving only the last line in eq.(68) multiplied by terms which are analytic in $\zeta$ around $\zeta=0$. Similarly we find

$$
\delta y_{2}(u) \approx \frac{a \zeta^{1 / 4}+b \zeta^{3 / 4}}{w_{12}} I_{22}(-1)-\frac{c \zeta^{1 / 4}+d \zeta^{3 / 4}}{w_{12}} I_{12}(-1)
$$

Thus the perturbed functions near a $u=-1$ are given by $(1+F)\left(\begin{array}{l}y_{1} \\ y_{2}\end{array}\right)$ where

$$
F=\frac{1}{w_{12}}\left(\begin{array}{ll}
I_{12}(-1) & -I_{11}(-1) \\
I_{22}(-1) & -I_{12}(-1)
\end{array}\right) .
$$

We come now to the imposition of the $S U(1,1)$ nature of the perturbed monodromy matrix which is the necessary and sufficient condition for having monodromy around $u=-1$. Allowing for a multiplication by $(1+\delta K) K$ with

$$
K=\left(\begin{array}{cc}
\kappa^{-1} & 0 \\
0 & \kappa
\end{array}\right), \quad \delta K=\left(\begin{array}{cc}
-\delta \kappa & 0 \\
0 & \delta \kappa
\end{array}\right)
$$


being $K$ the unperturbed scale transformation which realized the monodromies at all singularities, we have around -1

$$
Y=(1+\delta K) K(1+F)\left(\begin{array}{l}
y_{1} \\
y_{2}
\end{array}\right)
$$

giving for the monodromy matrix

$$
M+[\delta K, M]+\left[K F K^{-1}, M\right]
$$

being $M$ the unperturbed monodromy matrix. Thus $\delta M$ is given by

$$
\delta M=[\delta K, M]+\left[K F K^{-1}, M\right] .
$$

Imposition of $\delta M_{21}=\overline{\delta M_{12}}$ gives

$$
\left.w_{12}(\delta \kappa+\delta \bar{\kappa})=I_{12}(-1)+\overline{I_{12}(-1)}+\frac{\overline{M_{11}(-1)}}{\overline{M_{12}(-1)}} \overline{\left(\kappa^{-2}\right.} \overline{I_{11}(-1)}+\kappa^{2} I_{22}(-1)\right)
$$

where we took into account the $S U(1,1)$ nature of $M$ and its tracelessness. Using without loss of generality $\kappa$ and $\delta \kappa$ real we have

$$
2 w_{12} \delta \kappa=I_{12}(-1)+\overline{I_{12}(-1)}+\frac{\overline{M_{11}(-1)}}{\overline{M_{12}(-1)}}\left(\kappa^{-2} \overline{I_{11}(-1)}+\kappa^{2} I_{22}(-1)\right) .
$$

We come now to the monodromy at $u_{1}$. It is cumbersome to impose the monodromy on a moving singularity even if in principle one can deal with it. It is much simpler to perform the following coordinate transformation

$$
u=r \frac{u_{1}+\varepsilon}{u_{1}}
$$

and set $t_{k}(r)=y_{k}(u)$. The function $t(r)$ obeys the following differential equation

$$
t^{\prime \prime}(r)+\left(Q_{1}(r)+q_{1}(r)\right) t(r)=0
$$

with

$$
\begin{aligned}
Q_{1}(r) & =\frac{h}{16} \frac{r+\beta}{r\left(r-u_{1}\right)(r+1)}+\frac{3}{16}\left(\frac{1}{\left(r-u_{1}\right)^{2}}+\frac{1}{r^{2}}+\frac{1}{(r+1)^{2}}\right. \\
& \left.-2 \frac{1}{\left(u_{1}+1\right)\left(r-u_{1}\right)}+2 \frac{1}{\left(u_{1}+1\right)(r+1)}\right)
\end{aligned}
$$

and

$$
\begin{aligned}
q_{1}(r) & =\varepsilon \frac{h(1+r)(\beta-1)+6\left(1+u_{1}\right)}{16 u_{1}\left(u_{1}-r\right)(1+r)^{3}} \\
& -\beta_{1} \frac{h}{16\left(u_{1}-r\right) r(r+1)} .
\end{aligned}
$$


Working with the functions $t_{k}(r)$ we have

$$
\delta t_{k}(r)=\frac{1}{w_{12}}\left[t_{1}(r) J_{k 2}(r)-t_{2}(r) J_{k 1}(r)\right]
$$

with

$$
J_{l k}(r)=\int_{0}^{r} t_{l}(x) t_{k}(x) q_{1}(x) d x
$$

and repeating the procedure explained above we reach the equation

$$
2 w_{12} \delta \kappa=J_{12}\left(u_{1}\right)+\overline{J_{12}\left(u_{1}\right)}+\frac{\overline{M_{11}\left(u_{1}\right)}}{\overline{M_{12}\left(u_{1}\right)}}\left(\kappa^{-2} \overline{J_{11}\left(u_{1}\right)}+\kappa^{2} J_{22}\left(u_{1}\right)\right)
$$

being $M\left(u_{1}\right)$ the unperturbed monodromy matrix at $u_{1}$. The $I_{j k}(-1)$ and $J_{j k}\left(u_{1}\right)$ have the structure

$$
I_{j k}(-1)=\varepsilon E_{j k}+\beta_{1} B_{j k}, \quad J_{j k}\left(u_{1}\right)=\varepsilon H_{j k}+\beta_{1} L_{j k} .
$$

Then equating the r.h.s. of eqs. (69) w4 wave, given $\varepsilon$, a system of two equations in the two unknown $\beta_{1}^{R}, \beta_{1}^{I}$. As eqs. (69) (74) contain both $\varepsilon$ and $\bar{\varepsilon}$, we see that $\beta$ will not be an holomorphic function of $\varepsilon$ even though we expect $\beta^{R}$ and $\beta^{I}$ to be real analytic functions of the two variables $\varepsilon^{R}$ and $\varepsilon^{I}$ for small $\varepsilon$. Now the main point is that at the solution of such system of equations the value of the r.h.s. of eq.(69) ( and thus of eq.(174) ) has to be real and positive. As we discussed is detail in Sec.5 reality is a general consequence of the nature of the transformation matrices while positivity derives from the fact that for $\varepsilon=0$ such ratios are positive.

\section{Deformation of the square}

In this section we shall apply the formalism developed in the previous section to treat in terms of quadratures the deformed square for any coupling but small deformation.

In this case we know both the unperturbed value of the accessory parameter i.e. $\beta=0$ and the explicity form of the unperturbed solutions $y_{k}$ given is Sec 6 .

With regard to the perturbation $q$ for the monodromy around -1 and $q_{1}$ for the monodromy around 1 we have

$$
\begin{aligned}
q(u) & =\varepsilon \frac{h(1-u)-12}{16(1+u)(1-u)^{3}}-\beta_{1} \frac{h}{16 u\left(1-u^{2}\right)} \\
q_{1}(r) & =\varepsilon \frac{-h(1+r)+12}{16(1-r)(1+r)^{3}}-\beta_{1} \frac{h}{16 r\left(1-r^{2}\right)}
\end{aligned}
$$

from which we have $q_{1}(r)=-q(-r)$. On the other hand for the unperturbed $Q$ we have

$$
Q_{1}(r)=Q(r)
$$


so that the unperturbed solutions are the same i.e. $y_{k}^{(1)}(r)=y_{k}(r)$. Taking into account that

$$
I_{11}(-1)=e^{\frac{i \pi}{2}} I_{11}(1), \quad I_{12}(-1)=-I_{12}(1), \quad I_{22}(-1)=e^{\frac{3 i \pi}{2}} I_{22}(1)
$$

and

$$
M_{11}(-1)=M_{11}(1), \quad M_{12}(-1)=e^{\frac{i \pi}{2}} M_{12}(1)
$$

we have from (69) and (74) $2 w_{12} \delta \kappa=0$ i.e.

$$
0=I_{12}(1)+\bar{I}_{12}(1)+\frac{\overline{M_{11}(1)}}{\overline{M_{12}(1)}}\left(\kappa^{-2} \overline{I_{11}(1)}+\kappa^{2} I_{22}(1)\right)
$$

which determines the change of the accessory parameter $\beta$ from the value 0 to the value $\beta_{1}$. It can be written in terms of the unperturbed solutions (59). The result $\delta \kappa=0$ is not unexpected as the deformations from the square for symmetry reasons must give the same value of $\kappa$ for $\varepsilon$ and $-\varepsilon$. Substituting the so obtained value $\beta_{1}$ in eq.(75) and computing the ensuing $\delta y_{k}$ as we did in Sec,4 we obtain the $\phi$ for the deformed square.

\section{$9 \quad$ Addition of weak sources}

In reference 22 the technique developed in Sec4 enabled the computation in terms of quadratures of the exact Green function on the sphere in the background generated by three point sources of arbitrary strength. Such a result also gave the semiclassical 4point function with three arbitrary charges and a small one in terms of quadratures of hypergeometric functions. Here we shall apply a similar technique to the computation of the "symmetric" Green function on the square in the background of one arbitrary charge or equivalently the conformal factor generated by an arbitrary charge and two small charges in symmetric positions. The result extends immediately to any symmetric distribution of weak charges. This is a different kind of perturbation on the exact solution eq. (65).

Starting from

$$
Q(u)=\frac{1-\lambda^{2}}{16\left(u^{2}-1\right)}+\frac{3}{16} \frac{\left(1+u^{2}\right)^{2}}{u^{2}\left(1-u^{2}\right)^{2}}
$$

the addition of a new singularity of strength $\varepsilon$ at $u=t$ introduces the perturbation

$$
q(u)=\varepsilon\left[\frac{\left(1-\lambda^{2}\right) \beta}{16 u\left(u^{2}-1\right)}+\frac{1}{(u-t)^{2}}+\frac{\beta_{t}}{2(u-t)}+\frac{\beta_{1}}{2(u-1)}+\frac{\beta_{-1}}{2(u+1)}\right] .
$$

The $\beta$ 's are related by the Fuchs conditions

$$
\beta_{1}+\beta_{-1}+\beta_{t}=0, \quad 2+\beta_{1}-\beta_{-1}+t \beta_{t}=0 .
$$


the second one needed to leave the source at $z=0$ unchanged. It will be useful to choose as the independent parameters $\beta$ and $\beta_{t}$. For the changes in the solutions $y_{j}(u)$ we have as before

$$
\delta y_{j}(u)=y_{1}(u) \frac{I_{j 2}(u)}{w_{12}}-y_{2}(u) \frac{I_{j 1}(u)}{w_{12}}
$$

with

$$
I_{j k}(u)=\int_{0}^{u} q(x) y_{j}(x) y_{k}(x) d x .
$$

We shall examine first the monodromy at the new singular point $t$. For a tour around $t$ we have

$$
\delta I_{k j}(u)=\varepsilon \oint_{t} y_{k}(x) y_{j}(x)\left[\frac{\beta_{t}}{2(x-t)}+\frac{1}{(x-t)^{2}}\right] d x=i \pi \varepsilon\left[\beta_{t} y_{k}(x) y_{j}(x)+2\left(y_{k}(x) y_{j}(x)\right)^{\prime}\right]_{x=t}
$$

as the contribution of the remainder of $q(u)$ is zero, being such terms analytic and thus the monodromy matrix at $t$ becomes

$$
\left(\begin{array}{cc}
1+\frac{\delta I_{12}(t)}{w_{12}} & -\frac{\delta I_{11}(t)}{w_{12}} \\
\frac{\delta I_{22}(t)}{w_{12}} & 1-\frac{\delta I_{12}(t)}{w_{12}}
\end{array}\right) .
$$

For the computation of the change in the monodromies at 1 and -1 the procedure is very similar to the one explained in Sec 4 for the monodromy at -1 .

As we have discussed in Sec 5 a necessary condition for the realization of monodromy at $-1,0,1, t$ is

$$
\frac{M_{12}(-1)}{\bar{M}_{21}(-1)}=\frac{M_{12}(1)}{\bar{M}_{21}(1)}=\frac{M_{12}(t)}{\bar{M}_{21}(t)}
$$

and we can spend the two complex parameters $\beta, \beta_{t}$ to solve them. The explicit form of such equation is given below in eqs.(81,82). We perform now the usual transformation $N(n)=(1+\delta K) K M(n)$, where $n=-1,1, t$ with

$$
K=\left(\begin{array}{cc}
\kappa^{-1} & 0 \\
0 & \kappa
\end{array}\right), \quad \delta K=\left(\begin{array}{cc}
-\delta \kappa & 0 \\
0 & \delta \kappa
\end{array}\right)
$$

to reduce the three ratios in eq.(178) to modulus 1 .

We have now to prove that the ensuing transformation matrices are all $S U(1,1)$. Due to the choice of the canonical solutions at 0 the transformation in 0 is always

$$
D=\left(\begin{array}{cc}
i & 0 \\
0 & -i
\end{array}\right)
$$

In addition we always have

$$
N(-1) D N(1)=N(\infty) N(t)^{-1}
$$


The transformation $N(\infty)$ is an elliptic $S L(2, C)$ transformation and also $N(\infty) N^{-1}(t)$ is elliptic, being $N(t)$ infinitesimally near to the identity. Thus we have

$$
\operatorname{tr}(N(-1) D N(1))=2 \cos \gamma=\text { real. }
$$

The form of the new matrices $N(-1)$ and $N(1)$ taking into account their tracelessness is

$$
N(-1)=\left(\begin{array}{cc}
m & a e^{i \alpha} \\
\bar{a} & -m
\end{array}\right), \quad N(1)=\left(\begin{array}{cc}
n & b e^{i \alpha} \\
\bar{b} & -n
\end{array}\right),
$$

so that eq.(80) becomes

$$
2 e^{i \alpha} \operatorname{Im}(a \bar{b})=2 \cos \gamma
$$

which implies $e^{i \alpha}= \pm 1$. Due to the continuity of the perturbation we have $e^{i \alpha}=1$.

As a consequence of the general results of Sec 5 we have that $m$ and $n$ are pure imaginary, which assures the $S U(1,1)$ nature of $N(-1)$ and $N(1)$. With regard to $N(t)$ we have at the values $\beta, \beta_{t}$ for which eqs.(78) is satisfied

$$
\frac{N_{12}(t)}{\bar{N}_{21}(t)}=\frac{1}{\kappa^{2} \bar{\kappa}^{2}} \frac{\beta_{t} y_{1}^{2}(t)+2\left(y_{1}^{2}(t)\right)^{\prime}}{\bar{\beta}_{t} \bar{y}_{2}^{2}(t)+2\left(\bar{y}_{2}^{2}(t)\right)^{\prime}}=1
$$

The last expression can also be written as

$$
\frac{\beta_{t} y_{1}^{2}(t)+2\left(y_{1}^{2}(t)\right)^{\prime}}{\bar{\beta}_{t} \bar{y}_{2}^{2}(t)+2\left(\bar{y}_{2}^{2}(t)\right)^{\prime}}=\frac{\left(\beta_{t} y_{1}(t) y_{2}(t)+2\left[y_{1}(t) y_{2}(t)\right]^{\prime}\right)^{2}-4 w_{12}^{2}}{\left|\bar{\beta}_{t} \bar{y}_{2}^{2}(t)+2\left(\bar{y}_{2}^{2}(t)\right)^{\prime}\right|^{2}}>0 .
$$

But then being $w_{12}$ real we also have

$$
\beta_{t} y_{1}(t) y_{2}(t)+2\left[y_{1}(t) y_{2}(t)\right]^{\prime}=\text { real }
$$

which combined with eq. (766]77) gives $N_{22}(t)=\bar{N}_{11}(t)$ i.e. $N(t) \in S U(1,1)$. It follows from the group property (79) that also $N(\infty) \in S U(1,1)$ and thus we have fulfilled the monodromy at all singularities.

The explicit form of the two complex equations (78) which determine $\beta$ and $\beta_{t}$ is

$$
\begin{gathered}
I_{12}(-1)+\bar{I}_{12}(-1)+\frac{\bar{M}_{11}(-1)}{\bar{M}_{21}(-1)}\left(\bar{\kappa}^{-2} \bar{I}_{11}(-1)+\kappa^{2} I_{22}(-1)\right)= \\
I_{12}(1)+\bar{I}_{12}(1)+\frac{\bar{M}_{11}(1)}{\bar{M}_{21}(1)}\left(\bar{\kappa}^{-2} \bar{I}_{11}(1)+\kappa^{2} I_{22}(1)\right) \\
\bar{\kappa}^{2} \kappa^{2}=\frac{\beta_{t} y_{1}^{2}(t)+2\left(y_{1}^{2}(t)\right)^{\prime}}{\bar{\beta}_{t} \bar{y}_{2}^{2}(t)+2\left(\bar{y}_{2}^{2}(t)\right)^{\prime}}
\end{gathered}
$$

where $\kappa$ is the unperturbed parameter eq.(63) solving the problem of the square and $M( \pm 1)$ are the unperturbed transformation matrices of the $y_{k}(u)$ of Sec.6. 
Notice how eq.(82) already fixes the value of $\beta_{t}$ as it contains only the unperturbed parameter $\kappa$ and eq.(81) determines $\beta$. Finally

$$
I_{12}(1)+\bar{I}_{12}(1)+\frac{\bar{M}_{11}(1)}{\bar{M}_{21}(1)}\left(\bar{\kappa}^{-2} \bar{I}_{11}(1)+\kappa^{2} I_{22}(1)\right)=w_{12}(\delta \kappa+\delta \bar{\kappa})
$$

determines $\delta \kappa+\bar{\delta} \kappa$. We recall that $\kappa$ and $\delta \kappa$ can be chosen real.

We have with $\kappa_{1}=\kappa(1+\delta \kappa)$

$$
e^{-\phi / 2}=\frac{\kappa_{1}^{-2}\left(\bar{y}_{1}+\delta \bar{y}_{1}\right)\left(y_{1}+\delta y_{1}\right)-\kappa_{1}^{2}\left(\bar{y}_{2}+\delta \bar{y}_{2}\right)\left(y_{2}+\delta y_{2}\right)}{\sqrt{2}|\Pi(u)|^{2}}
$$

and taking the derivative with respect to $\varepsilon$ we have

$$
\begin{aligned}
& \left.-\frac{1}{8 \pi} \frac{\partial \phi}{\partial \varepsilon}=G(z, t)\right)= \\
& \frac{1}{4 \pi\left(\kappa^{-2} \bar{y}_{1} y_{1}-\kappa^{2} y_{2} y_{2}\right)}\left[\left(-2 \kappa^{\prime}+\frac{\mathcal{I}_{12}}{w_{12}}+\frac{\overline{\mathcal{I}}_{12}}{w_{12}}\right)\left(\kappa^{-2} \bar{y}_{1} y_{1}+\kappa^{2} \bar{y}_{2} y_{2}\right)-\right. \\
& \left.\bar{y}_{2} y_{1}\left(\kappa^{-2} \frac{\overline{\mathcal{I}}_{11}}{w_{12}}+\kappa^{2} \frac{\mathcal{I}_{22}}{w_{12}}\right)-y_{2} \bar{y}_{1}\left(\kappa^{-2} \frac{\mathcal{I}_{11}}{w_{12}}+\kappa^{2} \frac{\overline{\mathcal{I}}_{22}}{w_{12}}\right)\right]
\end{aligned}
$$

where

$$
\mathcal{I}_{j k}=\int_{0}^{u} \frac{q(x)}{\varepsilon} y_{j}(x) y_{k}(x) d x \quad \text { and } \quad \kappa^{\prime}=\frac{\delta \kappa}{\varepsilon} .
$$

This is the exact symmetric Green function in the background generated by the one point source of arbitrary strength on the square, given in terms of quadratures. It satisfies the equation

$$
\left(\Delta-e^{\phi}\right) G(z, t)=\delta^{2}\left(z-z_{t}\right)+\delta^{2}\left(z+z_{t}\right)
$$

being $\phi$ the background generated by the one point source placed at $z=0$ and $z_{t}$ is the image on the $z$-plane of $t$. It is possible to verify explicitely eq. (85) by taking the derivatives of expression (84) and using the relations

$$
I_{j k}^{\prime}(u)=q(u) y_{j}(u) y_{k}(u)
$$

$G$ has a singularity in $z= \pm z_{t}$ but it is regular at $z=0$ which is the position of the source as in happens in the sphere topology [22]. The quantity $e^{\phi}$ with $\phi$ given by eq.(83) is the semiclassical two point function with a source of finite strength and the other two of small strength.

\section{Conclusions}

In this paper we have applied in a systematic way the Riemann-Hilbert technique to Liouville theory on the torus. After a change of coordinates the problem is reduced to 
a Fuchsian differential equation. In the case of the one point function such differential equation is a particular case of the Heun differential equation, i.e. a differential equation with four singularities, which as well known, contains an accessory parameter which is not fixed by the Fuchs conditions. Then the main problem is to determine the value of such parameter. This is performed here in a few cases, through a perturbative technique, while in the case of the square the problem can be solved exactly.

The transformation through the Weierstrass function $\wp(z)=u$ gives a double valued representation of the torus in the $u$-plane. In the present paper we have examined situations which are symmetrical, where is not necessary to distinguish among the two sheets. We shall examine in an other paper the asymmetric situation.

The case of the one point function on the square is explicitely solvable through hypergeometric functions. The perturbative cases treated in the present paper are given by one weak source, by a general deformation and by the addition of a weak symmetric distribution of charges. Formulas for a general deformation are derived and then applied explicitely to the deformation of the square. An other application of the perturbative technique is the determination, through quadratures, of the exact symmetric Green function on the background generated by the one point source of arbitrary strength. The technique developed here can be applied to the semiclassical expansion of quantum Liouville theory on the torus.

\section{Acknowledgments}

I am grateful to Massimo Porrati for arising my interest in the problem and for useful discussions.

\section{References}

[1] E. Picard, Compt. Rend. 116 (1893) 1015; J. Math. Pures Appl. 4 (1893) 273 and (1898) 313; Bull. Sci. math. XXIV 1 (1900) 196

[2] H. Poincaré, J. Math. Pures Appl. (5) 4 (1898) 137

[3] L. Lichtenstein, Acta Mathematica 40 (1915) 1

[4] M. Troyanov, Trans. Am. Math. Soc. 324 (1991) 793

[5] A. Bilal, J-L. Gervais, J. Geom. Phys. 5 (1988) 277; Nucl. Phys. B305 (1988) 33. 
[6] A.B. Zamolodchikov, Al.B. Zamolodchikov, Nucl. Phys. B477 (1996) 577, e-Print: hep-th/9506136

[7] A.B. Zamolodchikov, Al.B. Zamolodchikov, e-Print: hep-th/0101152

[8] V. Fateev, A.B. Zamolodchikov, Al.B. Zamolodchikov, e-Print: hep-th/0001012

[9] H. Dorn, H.J. Otto, Nucl. Phys. B429 (1994) 375, e-Print: hep-th/9403141

[10] J. Teschner, Class. Quant. Grav.18 (2001) R153, e-Print: hep-th/0104158; Int. J. Mod. Phys. A19S2 (2004) 436, e-Print: hep-th/0303150; Phys. Lett. B363 (1995) 65, e-Print: hep-th/9507109

[11] Y. Nakayama, Int. J. Mod.Phys. A19 (2004) 2771, e-Print: hep-th/0402009

[12] V.A. Fateev, A.V. Litvinov, A. Neveu, E. Onofri, J. Phys. A42:304011 (2009), e-Print: arXiv:0902.1331 [hep-th]

[13] V.A. Fateev, A.V. Litvinov, Theor. Math. Phys. 154 (2008) 454, e-Print: arXiv:0707.1664 [hep-th]

[14] L. Hadasz, Z. Jaskolski, P. Suchanek, JHEP 1006:046 (2010), e-Print: arXiv:1004.1841 [hep-th]

[15] M. Flohr, M.R. Gaberdiel, J.Phys.A39 (2006) 1955, e-Print: hep-th/0509075

[16] R. Poghossian , JHEP 0912:038 (2009), e-Print: arXiv:0909.3412 [hep-th]

[17] V. Alba, A. Morozov, e-Print: arXiv:0911.0363 [hep-th]

[18] M. R. Gaberdiel, S. Lang, e-Print: arXiv:0810.0106 [hep-th]

[19] P. Olesen, Phys. Lett. B265 (1991) 361 and Phys. Lett. B268 (1991) 389

[20] N. Akerblom, G. Cornelissen, G. Stavenga, J.-W. van Holten, e-Print: arXiv:0912.0718 [hep-th]

[21] G. Felder, R Silvotti, Comm. Math. Phys. 123 (1989) 1

[22] P. Menotti, G. Vajente, Nucl. Phys. B709 (2005) 465, e-Print: hep-th/0411003

[23] P. Menotti, E. Tonni, Nucl. Phys. B707 (2005) 321, e-Print: hep-th/0406014 Phys. Lett. B633 (2006) 404, e-Print: hep-th/0508240 JHEP 0606:020 (2006), e-Print: hep-th/0602206 JHEP 0606:022 (2006), e-Print: hep-th/0602221; Proceedings of 
the 11th Marcel Grossmann Meeting on Recent Developments in Theoretical and Experimental General Relativity, Gravitation, and Relativistic Field Theories, Berlin, Germany, 23-29 Jul 2006. World Scientific, Singapore, 2008, pag.2647, e-Print: hep-th/0612107

[24] P. Menotti, J. Phys. Conf. Ser.33 (2006) 26, e-Print: hep-th/0512281

[25] A. Erdelyi (Ed.) "Higher Transcendental Functions", vol.II McGraw-Hill, New York, 1953

[26] E.T. Whittaker and G.N. Watson, "A Course of Modern Analysis", Chapt. XX, Cambridge University Press 1952

[27] A. Erdelyi, Duke Math. J. 9 (1942) 48; J. London Math. Soc. (1948) s1-23, 64

[28] R.S. Maier, Mathematics of Computation, 76 (2007) 811

[29] S. Yu. Slavyanov, and W. Lay "Special Functions: A Unified Theory Based on Singularities", Oxford University Press, 2000

[30] D.A. Hejhal, Acta Mathematica 135 (1975) 1

[31] A.A. Bolibrukh, Uspekhi Mat. Nauk 45 (1990) 3

[32] L. Cantini, P. Menotti, D. Seminara, Phys.Lett.B517 (2001) 203, e-Print: hep-th/0105081; Nucl. Phys. B638 (2002) 351, e-Print: hep-th/0203103

[33] J. A. Hempel, Bull. London Math. Soc. 20 (1988) 97; S. J. Smith, J. A. Hempel, J. London Math. Soc. (2) 40 (1989) 269; Bull. Austral. Math. Soc. 39 (1989), no. 3, 369

[34] C. Itzykson, J.B. Zuber, Nucl. Phys. B275 [FS17] (1986) 580

[35] P. Di Francesco, H. Saleur , J.B. Zuber, Nucl. Phys. B290 [FS20] (1987) 527

[36] A. Weil, "Elliptic functions according to Eisenstein and Kronecker", Spinger-Verlag, Berlin Heidelberg New York 1976

[37] M. Redi, Phys. Rev. D71:044006 (2005), e-Print: hep-th/0412189 